\title{
ATOMISTIC MODELING OF THE CRITICAL REGION OF COPPER USING A LIQUID-VAPOR COEXISTENCE CURVE
}

\author{
M.M. DEMIN, O.N. KOROLEVA* A.V. SHAPRANOV, A.A. ALEKSASHKINA \\ Keldysh Institute of Applied Mathematics of RAS, Russia, Moscow \\ * Corresponding author. E-mail: koroleva.on@mail.ru
}

DOI: $10.20948 /$ mathmontis-2019-46-6

Summary. A liquid - vapor coexistence curve was obtained for copper by the method of molecular dynamics modeling (MDM), and the corresponding critical parameters were determined: temperature, density, and pressure. The interaction potential of particles was of the "embedded atom" type (EAM). The critical temperature $T_{c r}$ was determined from the MDM results using the average cluster size method in the critical region. To clarify the critical density value, the empirical rule of rectilinear diameter was used. The results of modeling of this work were compared with the results of evaluating of the critical parameters of copper by other authors using different approaches.

\section{INTRODUCTION}

Studying the properties of metals in the vicinity of a critical point is a very important but difficult task. The critical point parameters are the most important characteristics of a substance [1], which in a generalized quantitative form express the effect of the action of intermolecular forces. Of particular interest is the boundary curve (binodal), which is the line of phase equilibrium of the liquid and gaseous phases and separates the homogeneous states of matter from two-phase metastable states. The metastable states of superheated liquid and saturated vapor have been studied relatively little [2]. Meanwhile, knowledge of the properties of superheated liquid and saturated vapor is required to calculate many practical problems [3]. In particular, the properties of the superheated liquid significantly affect the nature of the boiling of the liquid, and the properties of the supersaturated vapor determine the condensation process [4]. At the critical point, unlike other points of the boundary curve, the properties of both phases (liquid, vapor) are identical, that is, the critical state is the same limiting physical state of the substance for the both phases.

When approaching the critical point, the properties of a substance change, as new phenomena and mechanisms of interaction of particles of the substance appear, such as the fluctuations in the parameters of the substance (primarily density), the value of which grows very rapidly when approaching the critical point and tends to infinity at the critical point itself. In such a situation, consideration of the critical state on the basis of a theoretical approach using thermodynamic functions is applicable only in the region where fluctuations are relatively small [5].

The possibilities of experimental research are also limited. Due to the difficulty of conducting experiments at high temperatures, the critical point and binodal parameters were obtained only for a small amount of substances, which include alkali metals and mercury, which have relatively low temperature characteristics [6-11]. For other materials, there are

2010 Mathematics Subject Classification: $82 \mathrm{C} 26,74 \mathrm{~A} 15,74 \mathrm{~A} 25$.

Key words and Phrases: Molecular Dynamics Simulation, Near-Critical Region, Liquid-Vapor Coexistence Curve. 
only theoretical estimates within the framework of various models, among which phenomenological methods [12-24] and atomistic modeling methods [25-31] stand out.

The empirical relations connecting the parameters of the critical point with various characteristics of the substance in the liquid and gas state are widely used. These include the methods based on similarity laws connecting characteristic lines (for example, Zeno-line the unit compressibility line) of gases and liquids with critical parameters have also gained widespread popularity [14-17, 21,24] and the method of rectilinear diameter [5], estimates of critical parameters based on the relationship of metal vapors with the ionization potential of atoms [12], among these relations there is also the Kopp-Lang rule [13], which relates the critical temperature to the energy of evaporation. Estimates obtained from semiempirical equations of state are also widely used [20,22, 23]. In [18], a method was proposed for calculating the parameters of critical points and the binodal of a vapor - liquid (dielectric metal) transition in metal vapors. The model is based on the assumption that cohesion, which determines the basic characteristics of metals under normal conditions, is also responsible for the properties of metals in the vicinity of the critical point.

An important tool in the modeling of the properties of substances and physical processes inaccessible to direct measurement was mathematical modeling based on atomistic models. The atomistic approach is represented by Monte Carlo [19,29,30,31] and molecular dynamics [25-28] methods. Within the framework of the Monte Carlo method, to determine the critical parameters of metals and nonmetallic substances, the Wang - Landau (EWL) approach [29].

Mathematical modeling based on the molecular dynamics method over the past two decades has become a powerful tool for fundamental research of properties [25-27] and processes [28] in materials.

Despite the large number of estimated theoretical approaches, the obtained values of the critical parameters and binodal of copper have large differences. For example, in [29, 30] the critical parameters of copper were obtained from molecular dynamics simulations with the same potential (EAM), but using different techniques. The results obtained in these works for the critical temperature differ by 1.27 times. Despite the complexity of both theoretical and experimental approaches, interest in research in this area persists.

The aim of this work is to obtain a liquid - vapor coexistence curve for copper and determine the corresponding critical parameters: temperature, density, and pressure using molecular dynamics simulations with the EAM interaction potential [32]. This potential was previously tested and used to describe the processes in solid and liquid copper in the melting region in [33]. The calculations were performed using the LAMMPS package [34]

\section{STATEMENT OF THE PROBLEM}

\subsection{Mathematical formulation of the problem}

The molecular dynamics method is based on the representation of the considered object as a set of particles for which the Newton's equations are written. For each particle, the mass $m_{i}$, velocity $v_{i}$, and radius vector $r_{i}$ are considered. This results in a system of $2 \mathrm{~N}$ ordinary differential equations (ODE) 


$$
\left\{\begin{array}{l}
m_{i} \frac{d \vec{v}_{i}}{d t}=\vec{F}_{i}+F_{i}^{e x t} \\
\frac{d \vec{r}_{i}}{d t}=\vec{v}_{i} \quad, i=1 \ldots N
\end{array}\right.
$$

where $\vec{F}_{i}=-\frac{\partial U\left(\vec{r}_{1} \ldots \vec{r}_{N}\right)}{\partial \vec{r}_{i}}$ is the force of the interaction between the particles, $\vec{F}_{i}^{\text {ext }}$ is the force of interaction with the external fields, $U\left(\vec{r}_{1}, \ldots, \vec{r}_{N}\right)$ is the potential energy of the system of $N$ particles.

\subsection{Initial and boundary conditions}

At the initial moment of time, the object under consideration is a crystal at the temperature $T$. The particle velocities at the initial moment are set as random variables corresponding to the Maxwell distribution at twice the temperature 2T. Before the simulation starts, such an object is equilibrated with the thermostat [35] and barostat [36] turned on, and quite quickly part of the kinetic energy goes into potential one, and the temperature becomes $T$, and the pressure is set to $P=0$.

As boundary conditions, the periodic conditions are used. Under the periodic boundary conditions with respect to $O x$, it is believed that a particle exiting through the right boundary is replaced by a particle having the same velocity but entering through the left boundary.

Subsequently, the ODE system (1) is solved using the Verlet finite-difference scheme [37]. In this method, the coordinates of the particles are calculated on the integer time layers, and the velocity on half-integer ones.

\subsection{Particle interaction potential}

The accuracy of the results of molecular dynamics modeling substantially depends on the used particle interaction potential. Since the 1980s, the potentials of the "embedded atom" family (EAM) have become widespread for metals. To determine the branches of the liquid vapor coexistence curve and critical parameters of copper in this work, we used the EAM1 potential, which is a modification of the potential from this family, developed and tested on copper in [32]. The potential of EAM1 is based on both experimental and ab initio data. The results of testing of the potential on copper showed its reliability and good agreement with the experimental data.

\section{RESULTS AND DISCUSSION}

The computational domain was chosen in the form of a parallelepiped with sizes of $32 \times 15 \times 15$ unit cells of copper, which corresponds to a size of $11.568 \times 5.4225 \times 5.4225 \mathrm{~nm}^{3}$. This domain contains 29,250 particles. Periodic boundary conditions were set along all three axes. The dimensions of the region in which the sample is located are larger along the $\mathrm{x}$ axis than the dimensions of the sample. On this axis, the size occupied by the atoms of the sample were $11 \mathrm{~nm}$, and the size of the entire region was assumed to be $55 \mathrm{~nm}$. The selection of the size of the region was given special attention. Through some preliminary calculations, the 
sizes were determined so that the liquid - vapor boundary did not disappear as a result of evaporation before the time it reached the critical temperature. And vice versa, so that the entire area is not prematurely filled with an expanded fluid.

\subsection{Formation of the binodal branches}

The sample was heated using a thermostat by changing the current temperature $T$ by $\Delta T$. Relatively fast heating to a temperature of $4500 \mathrm{~K}$ in $100 \mathrm{ps}$ using a thermostat was carried out at zero pressure, supported by the switched-on barostat. This heating mode was maintained up to $5000 \mathrm{~K}$. After reaching $5000 \mathrm{~K}$, the barostat was turned off, since in the nearcritical region it can no longer maintain zero pressure. For this reason, the heating of the sample, by decreasing $\Delta T$, was slowed down, compared with heating with the barostat turned on, in order to avoid rupture of the liquid region. So, if, using the Berendsen thermostat, heating, starting from the temperature $T=4500 \mathrm{~K}$, was carried out with $\Delta T=500 \mathrm{~K}$, with $T=5000 \mathrm{~K}$ the $\Delta T$ value decreased to $250 \mathrm{~K}$, and with $T=6000 \mathrm{~K} \Delta T$ decreased to $100 \mathrm{~K}$.

The binodal branches were formed according to the results of MDM by averaging the density over time and space. In all calculations, after each temperature change, the system remained unchanged for a time $t=1 \mathrm{~ns}$. During this time, the density was averaged over time. Density was also averaged over space. We represent this procedure in the following example. Figure 1 shows the spatial density distributions of copper at two temperatures averaged at $6100 \mathrm{~K}$ and $6600 \mathrm{~K}$.

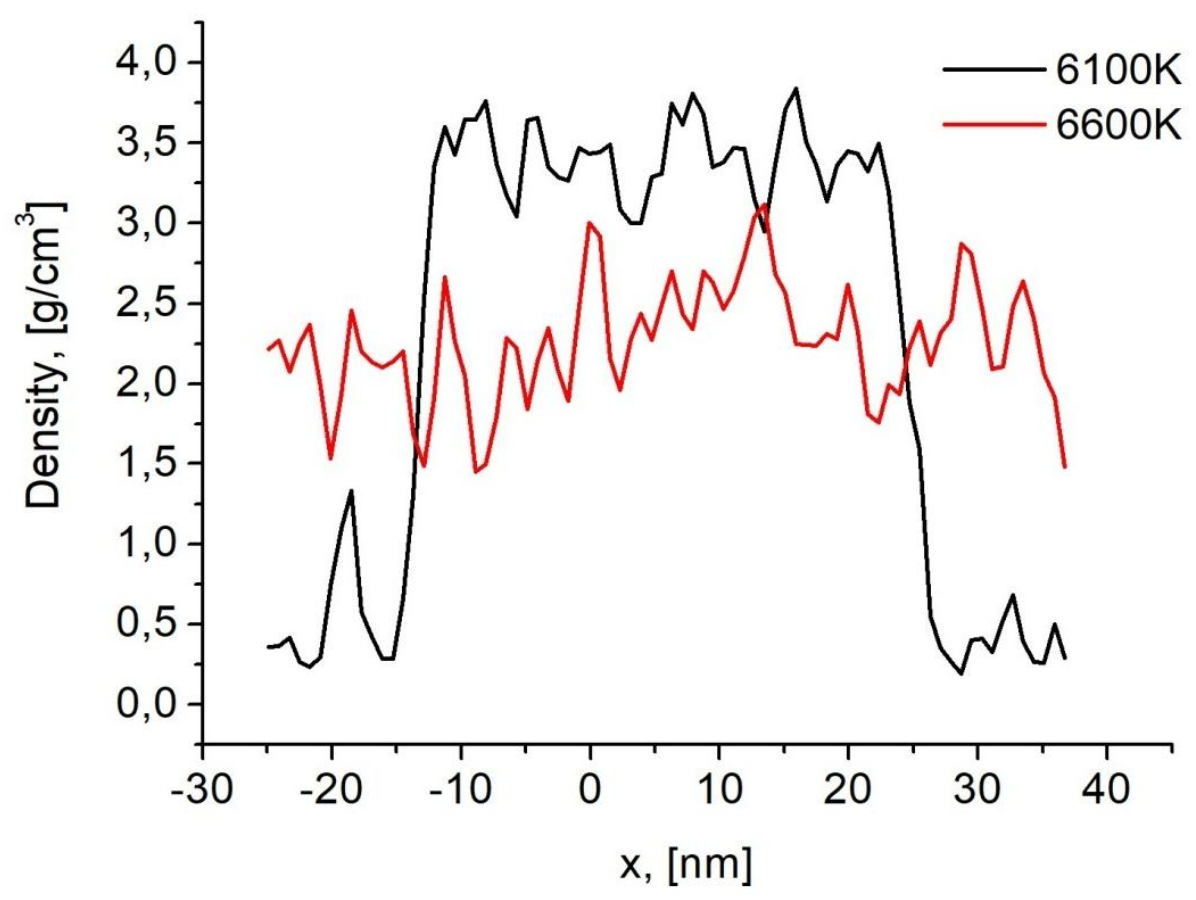

Fig. 1. The spatial distribution of the density of copper averaged over time.

The graph shows that for the temperature of $6100 \mathrm{~K}$, two spatial ranges $x_{1} \in(-25,-15) \mathrm{nm}$ and $x_{2} \in(-10,25) \mathrm{nm}$ are clearly distinguished, in which fluctuations around two different density values are noted. In each of these ranges, the coordinate $x$ was chosen in the middle 
where density was averaged over time. In the first range $x 1$, the average density $\bar{\rho}_{1}=0.397$

$\mathrm{g} / \mathrm{cm}^{3}$ was obtained, and in the second range $\mathrm{x}_{2}$, the average density $\bar{\rho}_{2}=3.5 \mathrm{~g} / \mathrm{cm}^{3}$. Both density values correspond to the same temperature. The first value belongs to the liquid branch, and the second to the vapor branch of the binodal at the temperature of $6100 \mathrm{~K}$. On the curve corresponding to the density of copper at the temperature of $6600 \mathrm{~K}$ (Fig. 1), the oscillations occur around one average value of $\bar{\rho} \approx 2.25 \mathrm{~g} / \mathrm{cm} 3$, which indicates an approximate equality of the density of the liquid and vapor of copper in this region, as well as the proximity of the indicated temperature of the substance $(T=6600 \mathrm{~K})$ to the critical temperature.

\subsection{Critical density $\rho_{c r}$}

The branches of the liquid-vapor phase equilibrium curve obtained by similar averaging were deposited on the $\rho-T$ plane (Fig. 2.). However, due to fluctuations, determining the exact value of the critical density is difficult. To clarify the value of the critical density, the rule of the rectilinear diameter is usually used [5]

$$
\rho_{L}+\rho_{v}=2 \rho_{c r}+\lambda\left(T_{c r}-T\right)
$$

where $\rho_{L}$ in the density of liquid at the temperature $T, \rho_{v}$ is the density of saturated vapor in equilibrium with the liquid phase at the same temperature. The coefficient $\lambda$ is different for different substances and is close to 1 . At $T \approx T_{c r}$ the rule of the rectilinear diameter has the form:

$$
\rho_{c r} \approx \frac{\rho_{L}+\rho_{v}}{2}
$$

The name "rectilinear diameter", is determined by the fact that in the coordinates $\rho-T$ the diameter of the curve $\rho(T)$ is a straight line. To draw the straight line, two points $D_{l}\left(T_{1}, \rho_{L v 1}\right)$ and $D_{2}\left(T 2, \rho_{L v 2}\right)$ are enough, the ordinates of which are the values calculated by formula (3) at a certain temperature. As the first point, the binodal values are selected, at a maximum temperature $T_{l}=6400 \mathrm{~K}$, which still allows the determination of density. For the temperature $T_{1}=6400 \mathrm{~K}$, the ordinate is calculated by formula (3) using the values of $\rho_{L}(6400)$ and $\rho_{v}(6400)$. To determine the second point $D_{2}\left(\mathrm{~T}_{2}, \rho_{\mathrm{Lv} 2}\right)$ of the rectilinear diameter, one can choose any of the values $\mathrm{T}<T_{1}$ and a pair of densities of the liquid and vapor branches of the binodal corresponding to this temperature. In total, five options were calculated for determining the coordinates of the second point, and five options were constructed for lines of rectilinear diameter passing through the point $D_{l}(6400 ; \rho(6400))$ and, therefore, five density values $\rho_{c r, i}(\mathrm{i}=0, . .4)$ can be obtained at the temperature $T_{c r}$, which has not yet been determined.

In fig. 2 rectilinear diameter options are shown with colored dashed lines.

The density value at the known value of $T=T_{c r}$ can be determined as the average

$$
\rho_{c r}=\sum_{i} \rho_{c r, i} / 5 \text {. }
$$




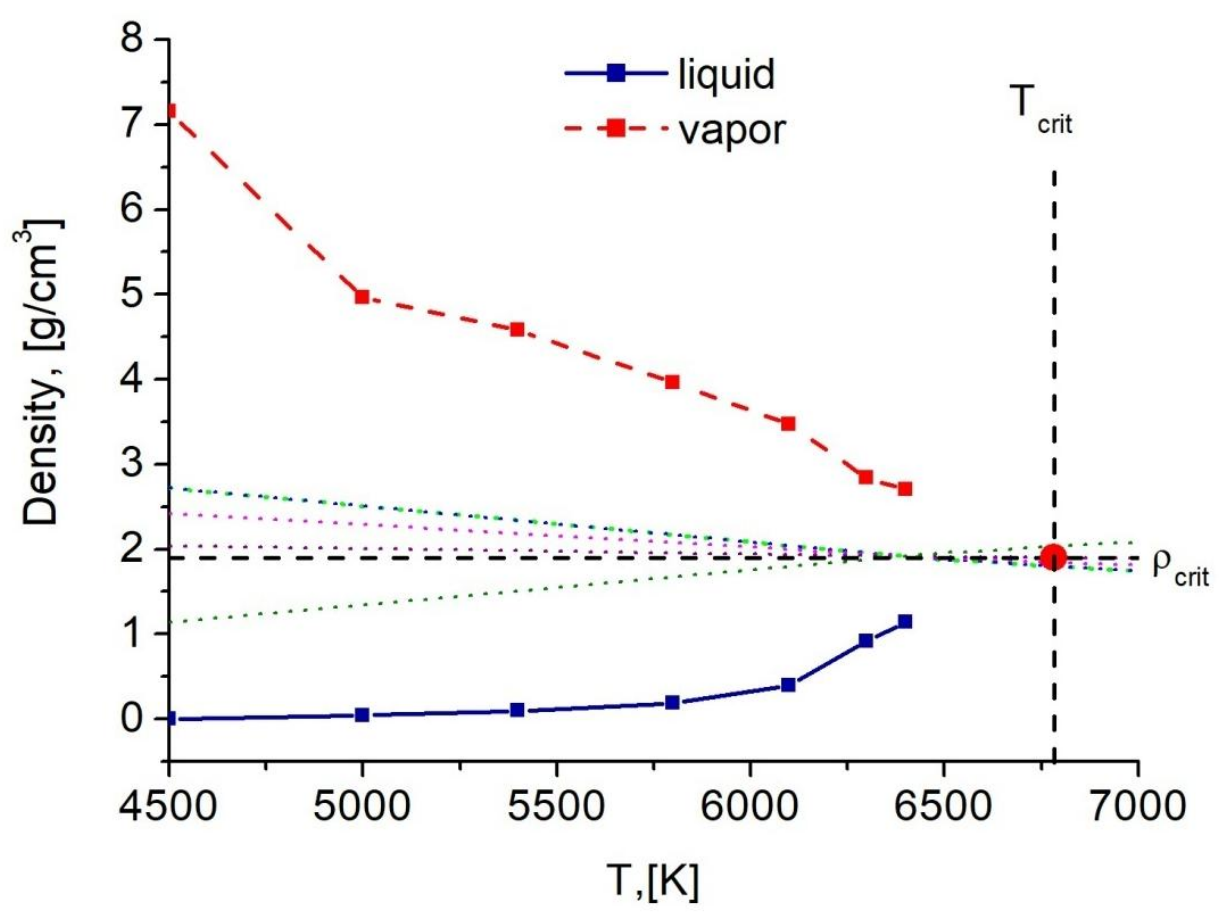

Fig.2. Binodal of copper.

\subsection{Critical temperature $T_{c r}$}

To obtain the critical density, it was necessary to determine the critical temperature $T_{c r}$, for which we used the method of the average cluster size in the critical region [38]. The essence of the method is as follows. In the subcritical region, with increasing temperature, the density and pressure of saturated vapor increase. In the near-critical region, atomic vapor particles begin to unite into clusters, which reach maximum sizes just at the critical point. With a further increase in temperature, the density no longer grows. Moreover, due to an increase in the kinetic energy of chaotic motion, the clusters begin to break up into smaller ones. Thus, the average cluster size must have a singularity at the critical point. This fact is used in this method to determine the critical temperature.

The average number of atomic particles forming a cluster can be estimated by the formula

$$
\langle N\rangle=\frac{n(T) k_{B} T}{P_{v}(T)},
$$

where $P_{v}(T)$ is the pressure of the saturated vapor at the given temperature $T, n(T)$ is the concentration of atomic particles in saturated vapor.

The temperature dependence of the average cluster size is shown in Fig. 3. The temperature plot has a characteristic kink at a critical point. Thus, using the average cluster size method, the critical temperature $T_{c r} \approx 6550 \mathrm{~K}$ was found. After that, the critical density $\rho_{c r} \approx 1.895 \mathrm{~g} / \mathrm{cm}^{3}$ was determined from (4). 


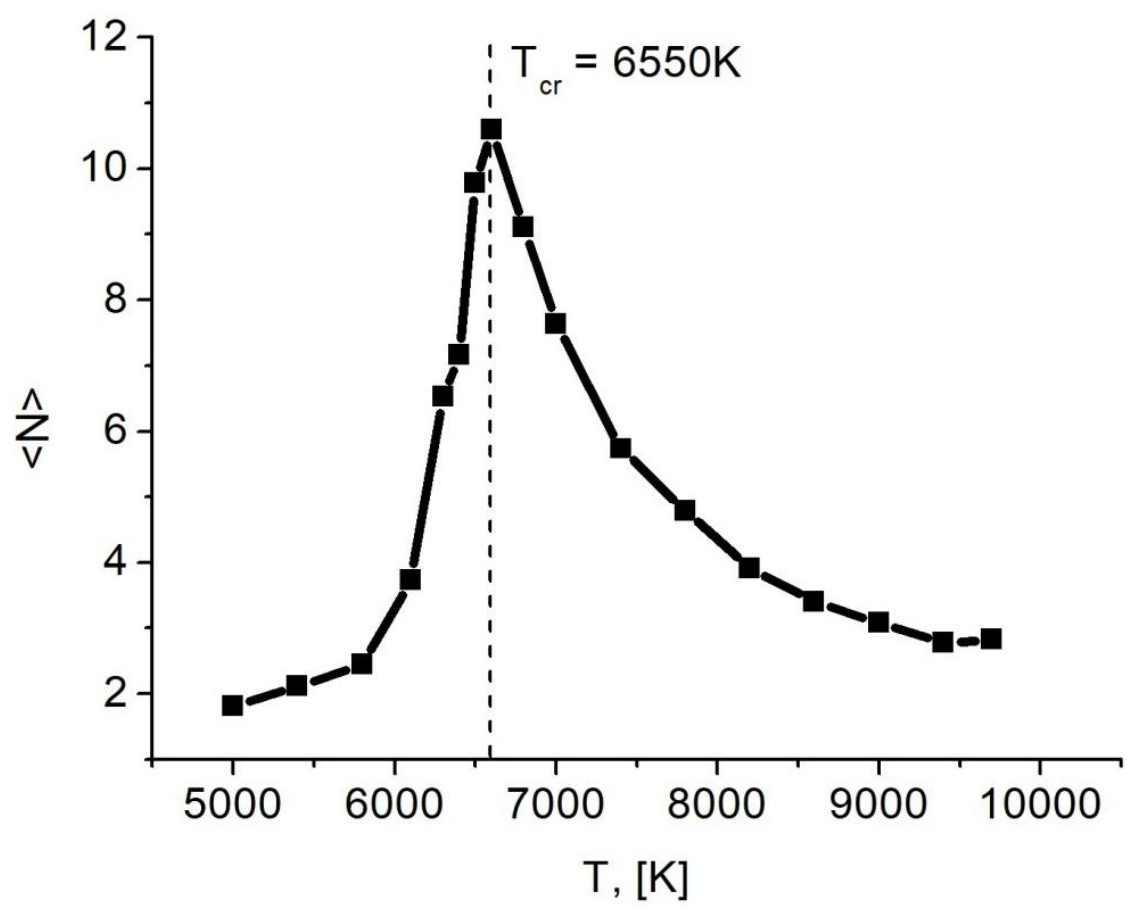

Fig.3. Temperature dependence of the cluster size.

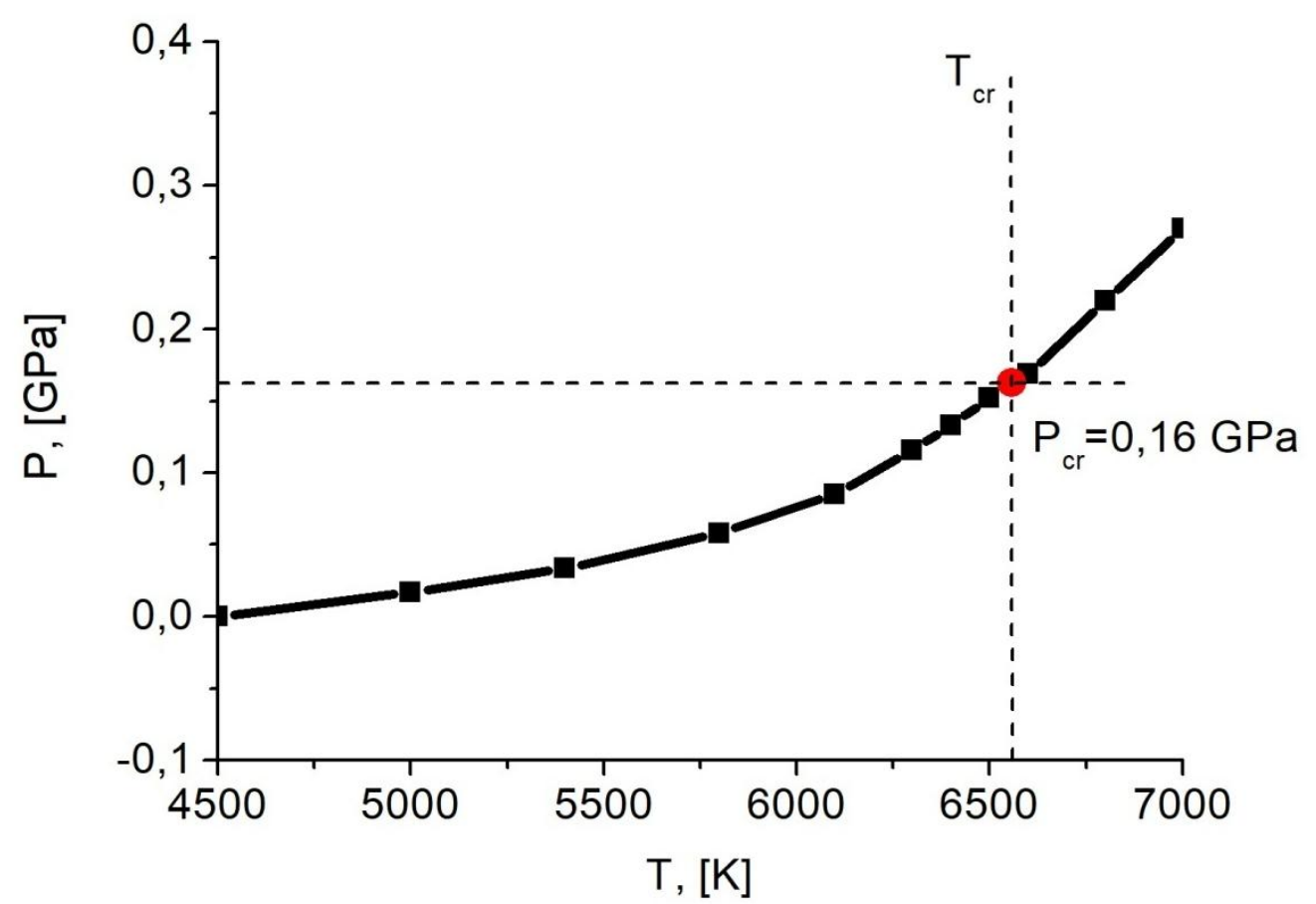

Fig. 4. Temperature dependence of saturated vapor pressure. 
The temperature dependence of the saturated vapor pressure $P_{v}$ was also obtained from the MDM results by averaging the results over time and space (Fig. 4). At the critical temperature $T_{c r}$ and density $\rho_{c r}$, the saturated vapor pressure $P_{v} \approx 0.16 \mathrm{GPa}$.

\subsection{Comparison of the results with the theoretical predictions}

Due to the lack of experimental information on the values of the critical parameters of copper, a comparison of the results was carried out with theoretical predictions of other studies. Table 1 shows the results of evaluating of the critical parameters of copper from this work and papers $[17,18,20-24,29,31]$. The critical parameter values in the table are shown in decreasing order of Tcr. Among them are estimates obtained: on the basis of similarity relations [17,21, 24]; derived from the semi-empirical equation of state [20,22,23]; using the concept of "cohesive energy" [18]; as a result of numerical simulation with Morse potential [31]; the potential of an embedded atom method [29]. The table also presents the results of calculating the relative deviation of the critical parameters obtained in this work from these results

$$
\sigma_{T c r}=\frac{T_{c r, N}-T_{c r, 10}}{T_{c r, N}} \quad \sigma_{\rho_{c r}}=\frac{\rho_{c r, N}-\rho_{c r, 10}}{\rho_{c r, N}} \quad \sigma_{P_{c r}}=\frac{P_{c r, N}-P_{c r, 10}}{P_{c r, N}}
$$

where $N=1, \ldots 9$ is the number of critical parameter values in the table $1, T_{c r, N}, \rho_{c r, N}, P_{c r, N}-$ are

\begin{tabular}{|c|c|c|c|c|c|c|c|}
\hline $\mathbf{N}$ & $\mathbf{T}_{\mathrm{cr}}, \mathbf{K}$ & $\sigma_{T_{c r}} \%$ & $\rho_{\mathrm{cr}}, \Gamma / \mathbf{c m}^{3}$ & $\sigma_{\rho_{c r}} \%$ & Pcr, ГПа & $\sigma_{P_{c r}} \%$ & References \\
\hline 1 & 8650 & 24,28 & 2.631 & 27,974 & 0.9543 & 83,234 & [31] \\
\hline 2 & 8440 & 22,39 & 1.94 & 2,32 & 0.651 & 75,422 & [20] \\
\hline 3 & 8390 & 21,93 & 2.39 & 20,71 & 0.746 & 78,552 & [21] \\
\hline 4 & 7850 & 16,56 & 2,63 & 0 & 0.905 & 82,32 & [22] \\
\hline 5 & 7625 & 14,10 & 1.058 & $-79,11$ & 0.83 & 80,72 & [23] \\
\hline 6 & 7620 & 14,04 & 1.4 & $-35,357$ & 0.577 & 72,27 & [24] \\
\hline 7 & 7580 & 13,59 & 1.58 & $-19,937$ & 0.7976 & 79,94 & [17] \\
\hline 8 & 7250 & 9,66 & 2.3 & 17,609 & 1.35 & 88,148 & [18] \\
\hline 9 & 5696 & $-14,99$ & 1.8 & $-5,278$ & 0.1141 & $-40,23$ & [29] \\
\hline 10 & 6550 & & 1.895 & & 0.16 & & This work \\
\hline
\end{tabular}
the critical parameters by other authors, for the present work $N=10$. The comparison results are shown at Figs. 5-7.

Table 1. The values of the parameters of copper at the critical point.

As can be seen from Table 1, the critical temperature and density obtained in this work are in good agreement with the results of other studies (Fig. 5, 6). The maximum deviation (less than $25 \%$ ) of the critical temperature obtained in this work from the results of [31] using the grandcanonical transition-matrix Monte Carlo method with the Morse potential. The smallest deviation (9.66\%) from the results of [18] (Fig. 5). The scatter of the deviation of the critical density values, as well as the $T_{c r}$ values, is small and amounts to $\approx 25 \%$. The exception is the results of $[23](\approx 80 \%)$ and $[24](\approx 35 \%)$ (Fig. 6). The critical pressure estimates differ quite significantly (Fig. 7). 


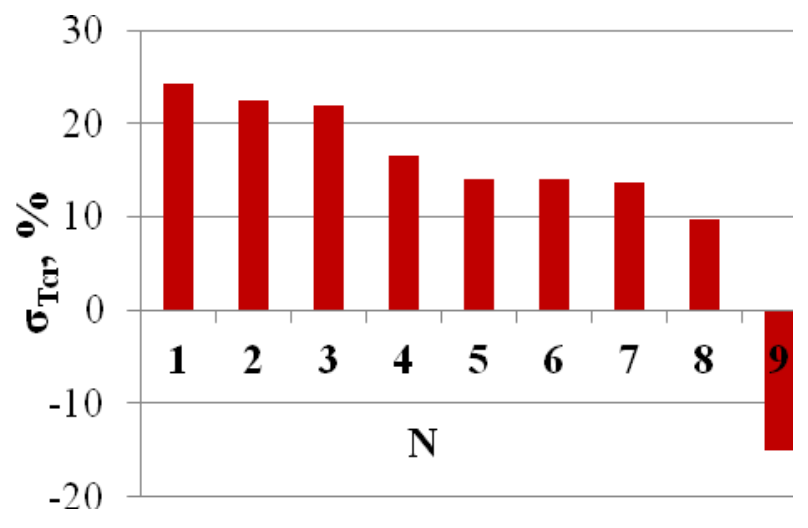

Fig. 5. Comparison results of $T_{c r}$ with other authors

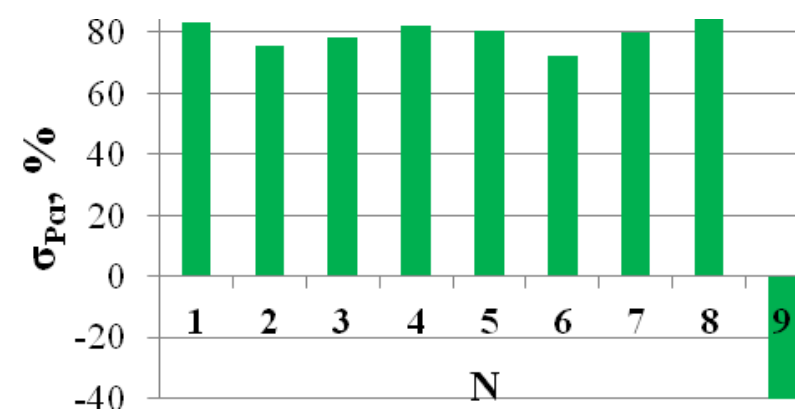

Рис. 7. Comparison results of $P_{c r}$ with other authors

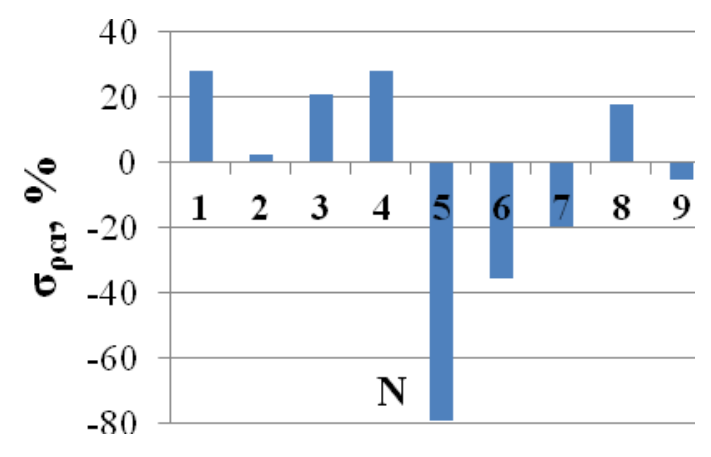

Рис. 6. Comparison results of $\rho_{c r}$ with other authors

In addition to [29], the deviation of the obtained $P_{c r}$ from other works is $\approx 80 \%$. This can be explained by the fact that pressure is the most sensitive characteristic of a substance that responds to any changes in the system.

The comparison of the obtained critical parameters with the results of [29], in which the interaction potential of particles from the family of the "embedded atom" (EAM) was used for modeling, showed the minimum differences of all critical parameters. Critical temperature differs by $15 \%$, critical density by $5 \%$, critical pressure by $40 \%$. Of all those shown in table 1 , these results are the closest. Obviously, this similarity of results is associated with the use of potentials of one family.

\section{CONCLUSION}

1. Based on a series of calculations using molecular dynamics modeling, a liquid - vapor coexistence curve was obtained and critical parameters of copper were determined: temperature, density, and pressure using the interaction potential of particles EAM1 [32].

2. The determination of the critical density value (according to the results of MDM) from the binodal curve due to strong density fluctuations near the critical temperature was carried out using the empirical rectilinear diameter procedure.

3. Значение критической температуры $T_{c r}$ определялось по результатам МДМ с использованием метода среднего размера кластеров в критической области. The critical temperature $T_{c r}$ was determined from the MDM results using the method of the average cluster size in the critical region.

4. A comparison of the simulation results of this work with the results of evaluations of the critical parameters of copper obtained by other researchers using different approaches showed a good coincidence. 


\section{REFERENCES}

[1] Shang-Keng Ma, Modern Theory of Critical Phenomena, Routledge, Taylor \& Francis Group, New York, (2018).

[2] V.P. Skripov. Metastabil naia zhidkost', M., Nauka, (1972).

[3] V.A. Kirilillin, V.V. Sychev, A.E. Sheindlin, Tekhnicheskaia termodinamika. M.: Izdatel`stvo ME'I, (2008).

[4] F. Hensel, "35 years Liquid Metals conferences: what do we and what do we not yet understand about liquid metals", J. Non-Cryst. Solids, 312-314, 1-7 (2002).

[5] M.P. Vukalovich, I.I. Novikov, Termodinamika, M.: Mashinostroenie, (1972).

[6] G. Franz, W. Freyland et F. Hensel, "Thermodynamic and electric transport properties of fluid cesium and rubidium in the M-NM transition region", J. Phys. Colloques, 41, C8-70 C8-73 (1980). DOI: 10.1051/jphyscol:1980819

[7] R. Winter, F. Hensel, T. Bodensteiner, W. Gläser, "The static structure factor of cesium over the whole liquid range up to the critical point", Ber. Bunsenges. Phys. Chem., 91(12), 13271330 (1987).

[8] R. Winter, C. Pilgrim, F. Hensel, C. Morkel, W. Gläser, "Structure and dynamics of expanded liquid alkali metals", J. Non-Crystalhne Sohds, 156-158, 9-14, (1993)

[9] F. Hensel, E. Marceca, W.C. Pilgrim, "The metal-non-metal transition in compressed metal vapours", J. Physics: Condensed Matter, 10(49), 11395-11404 (1998). doi: 10.1088/09538984/10/49/026

[10] F. Hensel, G.F. Hohl, D. Schaumloffel,W.C. Pilgrim, E.U. Franck, "Empirical regularities in behaviour of the critical constants of fluid alkali metals", Z. Phys. Chem., 214(6), 823-831 (2000).

[11] J. Jüngst, B. Knuth, F. Hensel, "Observation of singular diameter in the coexistence curve of metals", Phys. Rev. Lett., 55, 2160-2163 (1985).

[12] A.A. Likalter, "On the critical parameters of metals", High Temperature, 23(3), 371377 (1985).

[13] G. Lang, "Critical temperatures and temperature coefficients of the surface tension of liquid metals", Zeitschrift fuer Metallkunde, 68(3), 213-218 (1977)

[14] V.L. Kulinskii, "Simple Geometrical Interpretation of the Linear Character for the Zeno-Line and the Rectilinear Diameter", J. Phys. Chem. B, 114, 2852 (2010).

[15] V.L. Kulinskii, "The Critical Compressibility Factor Value: Associative Fluids and Liquid Alkali Metals”, J. Chem. Phys., 141, 054503 (2014).

[16] E.M. Apfelbaum, V.S. Vorob'ev, "The similarity relations set on the basis of symmetrization of the liquid - vapor phase diagram", J. Phys. Chem. B, 119, 8419 (2015).

[17] E.M. Apfelbaum and V.S. Vorob'ev, "The Wide-Range Method to Construct the Entire Coexistence Liquid-Gas Curve and to Determine the Critical Parameters of Metals", $J$. Phys. Chem. B, 119 (35), 11825-11832 (2015)

[18] A.L. Khomkin, A.S. Shumikhin, "Critical points of metal vapors". JETP, 121(3), 521- 528 (2015)

[19] C. Desgranges, J. Delhommelle, "Evaluation of the grand-canonical partition function using expanded Wang-Landau simulations. I. Thermodynamic properties in the bulk and at the liquid-vapor phase boundary", J. Chem. Phys., 136, 184107(1-12) (2012)

[20] A.A. Likalter, "Critical points of metals of three main groups and selected transition metals", Physica A: Statistical Mechanics and its Applications, 311,137-149 (2002)

[21] V.E Fortov, AN Dremin, AA Leont'ev, "Evaluation of the parameters of the critical point", High Temperature, 13(5), 984-992 (1975) 
[22] L.V. Al'tshuler, A.V. Bushman, M.V. Zhernocletov i dr., "Ize`ntropy` razgruzki i uravneniia sostoianiia metallov pri vy`sokikh plotnostiakh e`nergii”, ZHETF, 78(2), 741-760 (1980)

[23] D.A. Young, B.J. Alder, "Critial point of metals from the van der Waals model", Phys. Rev. A, 3(1), 364-371 (1971)

[24] A. A. Likalter, "Equation of state of metallic fluids near the critical point of phase transition", Phys. Rev. B, 53, 4386 (1996)

[25] V.I. Mazhukin, A.A. Samokhin, M.M. Demin, A.V. Shapranov, "Explosive boiling of metals upon irradiation by a nanosecond laser pulse", Quantum Electronics, , 44(4), 283-285 (2014)

[26] V.I. Mazhukin, A.A. Samokhin, A.V.Shapranov, M.M.Demin, "Modeling of thin film explosive boiling - surface evaporation and electron thermal conductivity effect", Mater. Res. Express, 2 (1), 016402 (1-9) (2015).

[27] Q.-L. Cao, P.-P. Wang, D.-H. Huang, Q. Li, F.-H. Wang, L. Cang Cai, "Pressure Dependence of Fusion Entropy and Fusion Volume of Six Metals", J. Chem. Eng. Data, 58(1), 64-70 (2013)

[28] M.V. Shugaev, C.Y. Shih, E.T. Karim, C. Wu, L.V.Zhigilei, "Generation of nanocrystalline surface layer in short pulse laser processing of metal targets under conditions of spatial confinement by solid or liquid overlayer", Applied Surface Science, 417, 54-63 (2017).

[29] T. Aleksandrov, C. Desgranges, J. Delhommelle, "Vapor-liquid equilibria of copper using hybrid Monte Carlo Wang-Landau simulations", Fluid Phase Equilib., 287, 79-83 (2010)

[30] E.M. Apfelbaum, V. S. Vorob'ev, "The Zeno line for Al, Cu, and U”, J. Phys. Chem. $B, 120,4828-4833$ (2016).

[31] J.K. Singh, J. Adhikari, S.K. Kwak, "Vapor-liquid phase coexistence curves for Morse fluids", Fluid Phase Equilib., 248, 1-6 (2006).

[32] Y. Mishin, M. J. Mehl and D. A. Papaconstantopoulos, A. F. Voter, J. D. Kress, "Structural stability and lattice defects in copper: Ab initio, tight-binding, and embedded-atom calculations", Phys. Rev. B, 63, 224106 (2001).

[33] V.I. Mazhukin, M.M Demin, A.A Aleksashkina, "Atomistic modeling of thermophysical properties of copper in the region of the melting point", Mathematica Montisnigri, 41, 99-111 (2018)

[34] S. Plimpton, "Fast parallel algorithms for short-range molecular dynamics", $J$. Comput. Phys., 117(1), 1-19 (1995).

[35] H.J.C. Berendsen, J.P.M Postma., W.F. van Gunsteren, A. DiNola, J.R. Haak, "Molecular dynamics with coupling to an external bath", J. Chem. Phys., 81, 3684 - 3690 (1984)

[36] M.P.Allen and D.J.Tildesley, Computer Simulation of Liquids, Oxford: Clarendon Press (2002)

[37] L. Verlet, "Computer "Experiments" on Classical Fluids. I. Thermodynamical Properties of Lennard-Jones Molecules", Phys. Rev., 159, 98-103 (1967).

[38] V.I. Mazhukin, A.V. Shapranov, O.N. Koroleva, A.V. Rudenko, "Molecular dynamics simulation of critical point parameters for silicon", Mathematica Montisnigri, 31, 56-76 (2014).

Received August 25, 2019 